\section{COMEPA (COVID-19 Medicina Policlinico Palermo): a study in hospitalized patients}

The COMEPA group*

Department of Internal Medicine and Geriatrics, University of Palermo, Italy

\section{Abstract}

Coronavirus disease 2019 (COVID-19) has dramatically changed our lives. In the past months, hospitals were saturated of patients; therefore, it is still important to have simple and standardized prognostic factors and to evaluate the efficacy and safety of medications commonly used for COVID-19. We aimed to collect data of the patients hospitalized in Internal Medicine and Geriatrics Wards at the University Hospital (Policlinico) 'P. Giaccone' in Palermo, Italy (COMEPA, COVID-19 Medicina Policlinico Palermo), with the main purpose of finding prognostic tools that can be easily used in clinical practice in order to identify patients hospitalized for/with COVID-19 at higher risk of negative outcomes, such as mortality, transfer to Intensive Care Unit (ICU) and institutionalization, as well as evaluating the efficacy/safety of medications commonly used for COVID-19. For reaching these aims, the medical records of approximately 600 patients will be recorded, having data on several parameters and including as outcomes mortality, ICU placement, institutionalization. With the COMEPA study, we therefore plan to update current literature, giving new data on prognostic factors and on the efficacy/safety of some medications used for COVID-19.

\section{Introduction}

In March 2020, the World Health Organization declared the coronavirus disease 2019 (COVID-19) outbreak as global pandemic. ${ }^{1}$ After more than one year of this outbreak that has dramatically changed our lives, more than 83 million people were affected by COVID-19, with over three million deaths in the world. ${ }^{2}$ All the National Health Systems faced the relevant problem of availability of hospital and Intensive Care Unit (ICU) beds during the COVID-19 epidemic. $^{3}$

For example, in the United States, sev- eral hospitals were saturated during the first two waves of COVID-19, particularly ICU beds. $^{4-6}$ In Italy, the COVID-19 outbreak caused similar issues, putting the hospitals and medical staff under psychological pressure. ${ }^{7}$ In Italy, different waves during 2020 and 2021 were present followed by an extensive amount of hospitalizations decreasing the resilience of the Italian healthcare system. ${ }^{8}$ The limited capacity of hospitals were among the most challenging issues facing authorities.

The limited amount of hospital beds, particularly in ICU, indicates the necessity of a precise stratification of patients following standardized prognostic factors. ${ }^{9}$ A living systematic review found several prognostic models prepared during COVID-19 outbreak, but all these models were rated at high or unclear risk of bias. ${ }^{10}$ In this sense, it would be helpful to have simple and inexpensive tools to identify the patient at higher risk of death and other relevant outcomes, such as ICU transfer and institutionalization. ${ }^{10}$

Given this background, we aimed to collect data of the patients hospitalized in Internal Medicine and Geriatrics Wards in the University Hospital (Policlinico) 'P. Giaccone' in Palermo, Italy (COMEPA, COVID-19 Medicina Policlinico Palermo), with the main purpose of finding prognostic tools that can be easily used in clinical practice in order to identify patients hospitalized for/with COVID-19 at higher risk of negative outcomes, such as mortality, transfer to ICU and institutionalization. Moreover, we will assess the efficacy/safety of some medications used for the empirical treatment of COVID-19 in real-life experience.

\section{Materials and Methods}

\section{Data source and inclusion/exclusion criteria}

All patients aging $>18$ years and hospitalized in Internal Medicine or Geriatrics Wards from $01^{\text {st }}$ September 2020 to $30^{\text {th }}$ April 2021 in the University Hospital (Policlinico) 'P. Giaccone' in Palermo, Sicily, Italy will be enrolled in this study. No other inclusion criteria will be proposed, to better represent real-life hospitalized people. The study was approved by the Local Ethical Committee during the session of the $28^{\text {th }}$ April 2021 (number 04/2021).

\section{Data collection}

The data from medical records will be recorded in a standardized Microsoft Excel file by resident doctors, supervised by experienced researchers.
Correspondence: Nicola Veronese, Geriatric Unit, Department of Internal Medicine and Geriatrics, University of Palermo, via del Vespro 141, 90127 Palermo, Italy.

Tel./Fax: +39.091.6554819.

E-mail: nicola.veronese@unipa.it

Key words: COMEPA; COVID-19; therapy; internal medicine; prognosis.

*The COMEPA group includes (alphabetical order): Affronti Marco, Amodeo Simona, Barbagallo Mario, Briganò Vincenza Maria, Cacioppo Federica, Capitano Walter M. Carruba Luca, Cavaleri Francesco, Catanese Giuseppina, Citarrella Roberto, Di Bella Giovanna, Di Franco Giuseppina, Di Prazza Agnese, Dominguez Ligia Juliana, Giannitrapani Lydia, Grasso Giulia, Immordino Federico, Licata Anna, La Carruba Anna, Mansueto Pasquale, Mirarchi Luigi, Morgante Maria Chiara, Parinello Alessandra, Pecoraro Emanuela, Peralta Marco, Polizzotto Carla, Pollicino Francesco, Quartetti Federico, Randazzo Giusi, Rizzo Angelo, Rizzo Giuseppina, Sanfilippo Valeria, Soresi Maurizio, Malerba Valentina, Vernuccio Laura, Veronese Nicola, Zerbo Maddalena.

Received for publication: 4 June 2021. Accepted for publication: 2 August 2021.

This work is licensed under a Creative Commons Attribution-NonCommercial 4.0 International License (CC BY-NC 4.0).

${ }^{\circ}$ Copyright: the Author(s), 2021

Licensee PAGEPress, Italy

Geriatric Care 2021; 7:9895

doi:10.4081/gc.2021.9895

\section{Statistical analyses}

Data on normality for the continuous variables will be assessed using the Kolmogorov-Smirnov test: if normality will be satisfied, data will be presented as means and standard deviation (SD) for quantitative measures or as median and interquartile range (IQR) if not normally distributed. For categorical variables, absolute numbers (and percentages) will be reported.

Depending on the outcome of interest, Cox's regression or logistic binary analysis will be used, including the assessment of potential covariates and confounders that should be associated with the outcome of interest having, at least, a -value $<0.10$. To test the robustness of our results, we plan to run sensitivity analyses (i.e., median age, gender and others pertinent), reporting the $\mathrm{p}$ for interactions. The propensity score will be applied in case of analyses regarding the analyses of medications, if necessary. ${ }^{11}$

All the analyses will be performed using the SPSS 21.0 for Windows (SPSS Inc., Chicago, IL, USA). All statistical tests 
will be two-tailed, setting a p-value $<0.05$ for the statistical significance.

\section{Results}

We plan to enroll all the patients admitted during the period mentioned before, for a total of approximately 600 hospitalized patients.

Table 1 shows the demographic and clinical information that we will record at the admission. Other than demographic information, data on physiological anamnesis will be collected. Moreover, we plan to register clinical information at the hospital admission also in terms of COVID-19 variants, onset of clinical symptomatology and signs/symptoms of COVID-19, and vital signs (e.g., body temperature, blood pressure, cardiac and respiratory frequency). We decided to record the presence and the severity of comorbidities using the cumulative illness rating scale (CIRS). ${ }^{12}$ The CIRS uses a 5-point ordinal scale (score 1-5) to estimate the severity of pathology in 13 different systems. Finally, we plan to record and analyze the data regarding bio-humoral and microbiological exams as well as the data regarding CT scans, as fully reported in Table 1.

Table 2 shows the information that we will record regarding therapy used during the hospitalization against COVID-19 infection. Therefore, the database will include information regarding oxygen therapy, corticosteroids, heparins, antibiotics (given by general practitioners and during the hospitalization), and remdesivir.

Finally, Table 3 illustrates the outcomes of our investigation that will be mortality (including the cause of death, if available from death certificates), clinical recovery, ICU and sub-ICU admission, and in case of older patients, institutionalization.

\section{Discussion and Conclusions}

With the COMEPA study, we aim to enroll all the patients hospitalized for and with COVID-19 infection during the last months in our wards of Internal Medicine and Geriatrics in Palermo, Italy. Overall, about 600 patients will be enrolled giving a picture of the clinical and bio-humoral characteristics of the patients present in our wards during 2020 and 2021, affected by this condition.

During the first wave (i.e., in the first six months of 2020), Sicily was relatively free from COVID-19 infection, as shown by national data. ${ }^{13}$ However, in the second part of 2020 and particularly during the first part of 2021, the problem of COVID-19 becomes very important in Sicily, particularly in the Palermo area. ${ }^{14}$ Therefore, to have clinical and epidemiological data specific for this area could be relevant, also in case of a new wave of this condition.

We would like to discuss some peculiar aspects of the COMEPA study. First, we will assess the clinical importance of the variants in this specific geographical area in which no data are available in terms of mortality or other negative outcomes, compared to the most frequent variant present in Italy. The presence of some variants, in fact, in Italy and in Sicily is exponentially increasing in these months. For example, the English variant (lineage B.1.1.7) seems to increase as prevalence in Sicily, ${ }^{15}$ even if

Table 1. The COMEPA study: demographic and general clinical information.

\begin{tabular}{|c|c|}
\hline Domain & Component \\
\hline Demographics & Age, gender, date of hospital admission \\
\hline Physiological anamnesis & Work (if any), smoking status, alcohol abuse, drug \\
\hline addition & \\
\hline Clinical information at the hospital admission & $\begin{array}{l}\text { Nasopharyngeal swab positive (date), presence of } \\
\text { variants, onset of the clinical symptomatology, signs } \\
\text { and symptoms of COVID-19, vital signs }\end{array}$ \\
\hline Comorbidity assessment & Cumulative illness rating scale \\
\hline Arterial blood gas analysis & $\mathrm{Ph}, \mathrm{pO}_{2}, \mathrm{pCO}_{2}$, lactates, $\mathrm{pO}_{2} / \mathrm{FiO}_{2}$ \\
\hline Bio-humoral exams & $\begin{array}{l}\text { Standard exams (e.g., complete blood count, renal } \\
\text { function), hsCRP, pro-calcitonin, total cholesterol } \\
\text { (and its components), serum 25OHD, proBNP, TSH }\end{array}$ \\
\hline Microbiological exams & $\begin{array}{l}\text { Urine and blood cultures, Legionella, pneumococcus } \\
\text { antigen, rectal swab }\end{array}$ \\
\hline CT exams & Brain, chest, abdomen \\
\hline
\end{tabular}

Table 2. The COMEPA study: information regarding therapy.

\begin{tabular}{ll} 
Domain & Details \\
Oxygen therapy & $\begin{array}{l}\text { Type, duration and quantity of oxygen administered } \\
\text { during the hospitalization }\end{array}$ \\
Corticosteroids & Type and duration \\
\hline Heparins & Type and duration \\
\hline Antibiotics & Before and during hospitalization \\
\hline Remdesivir & Duration \\
\hline
\end{tabular}

Table 3. The COMEPA study: outcomes.

\begin{tabular}{ll} 
Domain & Details \\
Mortality & Date and cause \\
Clinical recovery & Date \\
\hline ICU admission & Date and exitus \\
Sub intensive care unit admission & Date and exitus \\
\hline Institutionalization & Date \\
\hline
\end{tabular}

the prognostic value is still not entirely clear. ${ }^{16}$ Moreover, we plan to analyze not only the impact of the presence of some medical conditions, but also the severity using the CIRS. In this sense, very limited data are nowadays present, but they could important to better understand if some conditions are associated with a greater risk of mortality than others.

Furthermore, we will assess the impact of unidimensional (e.g., bio-humoral exams) and multidimensional scores for the prognosis in hospitalized patients affected by COVID-19. In this sense, several prognostic models were prepared during COVID-19 outbreak, but all these models were of moderate to poor quality, indicating the necessity of verifying these scores in other contexts and to find other tools for predicting prognosis. ${ }^{10}$ 
A relevant part of our work will be given to medications used during hospitalization. The topic of medications that could improve prognosis during COVID-19 infection is of critical importance. During these months several therapeutically approaches were tried, often without significant results. For example, with our experience we understand that oxygen should be given with positive pressure early to our patients in order to improve prognosis. ${ }^{17}$ However, some debates still remain in mild to moderate forms that are, on the contrary, frequently hospitalized. ${ }^{18}$ Similarly, we can deal with other medications commonly used for the treatment of COVID-19 including corticosteroids, ${ }^{19}$ heparins ${ }^{20}$ or antibiotics. Finally, since a consistent part of our patients was treated with remdesivir, we will assess the impact of this antiviral medication on the clinical course of COVID-19.21 For remdesivir, in fact, which is commonly used in our hospitals, a definitive word cannot be said in terms of efficacy and safety. At the same time, we believe that data regarding medications in observational studies are important since they represent real-life experience in which frailer people that are usually hospitalized but excluded by the randomized controlled trials, are represented.

In conclusion, with the COMEPA study, we would like to explore the importance of some prognostic factors and the efficacy/safety of some medications used for the empirical treatment of COVID-19 based on our real-life experience. We believe that our data could be important for better understanding some still not known aspects of this condition in hospitalized patients.

\section{References}

1. Jebril N. World Health Organization declared a pandemic public health menace: A systematic review of the coronavirus disease 2019 "COVID-19”, up to 26th March 2020. Available at SSRN 3566298; 2020.

2. Organization WH. Coronavirus disease 2019 (COVID-19): situation report, 45. 2020.

3. Haas LE, de Lange DW, van Dijk D, van Delden JJ. Should we deny ICU admission to the elderly? Ethical considerations in times of COVID-19. Critical Care 2020;24:1-3.

4. White DB, Lo B. A framework for rationing ventilators and critical care beds during the COVID-19 pandemic. JAMA 2020;323:1773-4.

5. Khullar D, Bond AM, Schpero WL. COVID-19 and the financial health of US hospitals. JAMA 2020;323:2127-8.

6. Birkmeyer JD, Barnato A, Birkmeyer $\mathrm{N}$, et al. The impact of the COVID-19 pandemic on hospital admissions in the United States: study examines trends in US hospital admissions during the COVID-19 pandemic. Health Affairs 2020;39:2010-7.

7. Chirico F, Nucera G, Magnavita N. Protecting the mental health of healthcare workers during the COVID-19 emergency. BJPsych Int 2021;18(1).

8. Kozyreff G. Hospitalization dynamics during the first COVID-19 pandemic wave: sir modelling compared to Belgium, France, Italy, Switzerland and New York City data. Infect Dis Model 2021;6:398-404.

9. Polidori MC, Maggi S, Mattace-Raso F, Pilotto A. The unavoidable costs of frailty: a geriatric perspective in the time of COVID-19. Geriatric Care 2020;6:8989.

10. Wynants L, Van Calster B, Collins GS, et al. Prediction models for diagnosis and prognosis of covid-19: systematic review and critical appraisal. BMJ 2020;369.

11. Guo S, Fraser MW. Propensity score analysis: Statistical methods and applications. Vol 11: SAGE publications; 2014.

12. Linn BS, Linn MW, Gurel L. Cumulative illness rating scale. J Am Geriatr Soc 1968;16:622-6.
13. Distante C, Piscitelli P, Miani A. Covid19 outbreak progression in Italian regions: approaching the peak by the end of March in northern Italy and first week of April in southern Italy. Int J Environ Res Public Health 2020;17:3025.

14. Sanità IS. COVID-19 integrated surveillance data in Italy; 2021. Available from: https://www.epicentro.iss.it/en/coronavirus/sars-cov-2-dashboard Accessed: 05/17/2021.

15. Davies NG, Jarvis CI, Edmunds WJ, et al. Increased mortality in communitytested cases of SARS-CoV-2 lineage B. 1.1. 7. Nature 2021:1-5.

16. Loconsole D, Sallustio A, Accogli M, et al. Outbreak investigation of symptomatic SARS-COV-2 VOC 202012/01lineage B. 1.1. 7 infection in healthcare workers, Italy. Clin Microbiol Infection 2021 [Epub ahead of print].

17. Winck J, Scala R. Non-invasive respiratory support paths in hospitalized patients with COVID-19: proposal of an algorithm. Pulmonology 2021 [Epub ahead of print].

18. Lechien JR, Chiesa-Estomba CM, Radulesco $\mathrm{T}$, et al. Clinical features of patients who had two COVID-19 episodes: a European multicentre case series. J Intern Med 2021 [Epub ahead of print].

19. Veronese N, Demurtas J, Yang L, et al. Use of corticosteroids in coronavirus disease 2019 pneumonia: a systematic review of the literature. Front Medicine 2020;7:170.

20. Bolzetta F, Maselli M, Formilan M, et al. Prophylactic or therapeutic doses of heparins for COVID-19 infection? A retrospective study. Aging Clin Exper Res 2021;33:213-7.

21. Okoli GN, Rabbani R, Copstein L, et al. Remdesivir for coronavirus disease 2019 (COVID-19): a systematic review with meta-analysis and trial sequential analysis of randomized controlled trials. Infect Dis 2021:1-9. 